\title{
Matrix metalloproteinase 9 may be involved in contraction of vascular smooth muscle cells in an in vitro rat model of subarachnoid hemorrhage
}

\author{
BAOQI DANG ${ }^{1,2^{*}}$, HAITAO SHEN $^{2 *},{\text { HAIYING } \mathrm{LI}^{2}, \text { MIN ZHU }^{1}, \mathrm{CHUNHUA} \mathrm{GUO}^{1} \text { and WEICHUN HE }}^{1,2}$ \\ ${ }^{1}$ Department of Neurosurgery, Zhangjiagang Hospital of Traditional Chinese Medicine, \\ Nanjing University of Chinese Medicine, Suzhou, Jiangsu 215600; ${ }^{2}$ Brain and Nerve Research Laboratory, \\ The First Affiliated Hospital of Soochow University, Suzhou, Jiangsu 215006, P.R. China
}

Received August 24, 2015; Accepted August 30, 2016

DOI: $10.3892 / \mathrm{mmr} .2016 .5736$

\begin{abstract}
Our previous study determined that prominent cerebral vasospasm (CVS) may occur in an in vivo model of subarachnoid hemorrhage $(\mathrm{SAH})$ in rats. Matrix metalloproteinase 9 (MMP-9) expression levels in basilar arteries were upregulated in a similar manner to the development of CVS following SAH. To identify the changes that occur in the contractility of cerebrovascular smooth muscle cells and the expression levels of MMP-9 in an in vitro model of $\mathrm{SAH}$, rat cerebrovascular smooth muscle cells were isolated, cultured, and then stimulated with hemolysate. Additionally, 2-[(4-phenoxyphenylsulfonyl)methyl]thiirane (SB-3CT), a selective MMP-9 inhibitor, was used to determine the effect of MMP-9 on the contractility of cerebrovascular smooth muscle cells. Cerebrovascular smooth muscle cells were successfully isolated and cultured in vitro, and hemolysate stimulation enhanced their contractility and increased MMP-9 expression levels. The present study also revealed that pretreatment with SB-3CT decreased MMP-9 expression levels in cerebrovascular smooth muscle cells, and reduced their contractility upon hemolysate treatment. Therefore, the current study confirmed that MMP-9 is important for the enhancement of the contractility of cerebrovascular smooth muscle cells in an in vitro rat model of SAH.
\end{abstract}

Correspondence to: Dr Weichun He, Department of Neurosurgery, Zhangjiagang Hospital of Traditional Chinese Medicine, Nanjing University of Chinese Medicine, 77 South Changan Road, Suzhou, Jiangsu 215600, P.R. China

E-mail: zhenjiangdbq@163.com

"Contributed equally

Abbreviations: CVS, cerebral vasospasm; MMP-9, matrix metalloproteinase 9; DMEM/F12, Dulbecco's modified eagle medium/Nutrient Mixture F-12; ROS, reactive oxygen species

Key words: matrix metalloproteinase 9, smooth muscle cell, cell contraction, cerebral vasospasm, subarachnoid hemorrhage

\section{Introduction}

The surgical and endovascular treatments of aneurysms and neurological intensive care have greatly improved. Therefore, the possibility of a hemorrhage following an aneurysm re-rupture is well-controlled. However, cerebral vasospasm (CVS) continues to be the primary reason for mortality and disability in patients with subarachnoid hemorrhage (SAH) (1). The pathogenesis of CVS remains to be fully elucidated. CVS may be due to various factors, including the increase of cerebral levels of NO, oxidative injury, platelet activation and aggregation, that enhance cerebral vasoconstriction and reduce cerebral vasodilation (2-5).

Matrix metalloproteinase 9 (MMP-9) is part of the gelatinase subfamily of MMPs and is expressed in various cells in the brain. Activated MMP-9 is responsible for the degradation of several proteins which constitute the extracellular matrix, including collagen types IV, V, VII and X, elastin, fibrillin, osteonectin and laminin. Therefore, MMP-9 is important for the pathogenesis of intracranial aneurysms (6-8). Our previous study determined that CVS may develop in rats following SAH (9). Additionally, MMP-9 expression levels in basilar arteries were upregulated with a time course similar to that of the development of CVS. This suggests that MMP-9 may be involved in the pathogenesis of CVS (9).

To the best of our knowledge, this is the first study that has aimed to examine the association between CVS and MMP-9. Therefore, the present study aimed to determine the underlying molecular mechanisms of CVS pathogenesis. The importance of MMP-9 for the contraction of cerebrovascular smooth muscle cells was also investigated.

\section{Materials and methods}

Isolation and culture of cerebrovascular smooth muscle cells. Four adult male Sprague-Dawley rats weighing 300-350 g were sacrificed by decapitation in each treatment group. A total of 12 rats were used in the present study, purchased from the Animal Center of Chinese Academy of Sciences (Shanghai, China). All animals were housed at a constant temperature of $22^{\circ} \mathrm{C}$, under a 12-h light/dark cycle (lights switched on at 6:00 a.m.) 
with free access to food and water. All rats were placed under general anesthesia prior to fixation-perfusion and euthanasia procedures. All procedures were approved by the Institutional Animal Care Committee of the Zhangjiagang Hospital of Traditional Chinese Medicine (Suzhou, China and were performed in accordance with the guidelines of the National Institutes of Health on the care and use of animals. Whole brains were dissected under sterile conditions and immediately placed in a culture dish with Dulbecco's modified Eagle's medium supplemented with Gibco ${ }^{\circledR}$ nutrient mixture F-12 (DMEM/F12), 100,000 U/1 penicillin and 100,000 U/1 streptomycin (Thermo Fisher Scientific, Inc. Waltham, MA, USA). Basilar arteries were dissected out in a laminar flow hood and rinsed repeatedly with DMEM/F12 medium supplemented with 100,000 U/1 penicillin and 100,000 U/1 streptomycin. (Thermo Fisher Scientific, Inc.). Following excision of the adventitial layer, the vessels were sectioned into small segments of length $\sim 0.2 \mathrm{~mm}$. The tissue was digested in $1 \mathrm{ml} 0.1 \%$ collagenase I solution (Sigma-Aldrich; Merck Millipore, Darmstadt, Germany) at $37^{\circ} \mathrm{C}$ with $5 \% \mathrm{CO}_{2}$ for $30 \mathrm{~min}$ until the tissue appeared swollen. In order to digest the tissue further, $1 \mathrm{ml}$ $0.125 \%$ trypsin solution (Gibco; Thermo Fisher Scientific, Inc.) was added for an additional $10 \mathrm{~min}$. The dispersed cells were collected and transferred to a centrifuge tube, and DMEM/F12 medium supplemented with $20 \%$ fetal bovine serum (FBS; Gibco; Thermo Fisher Scientific, Inc.) was added to terminate digestion. The cell suspension was subsequently centrifuged at $200 \mathrm{x} g$ for $5 \mathrm{~min}$, and the supernatant discarded. The cells were resuspended in DMEM/F12 medium with $20 \% \mathrm{FBS}$, seeded into a $60 \mathrm{~mm}$ culture dish and incubated at $37^{\circ} \mathrm{C}$ with $5 \% \mathrm{CO}_{2}$. The medium was changed every 3 days. After 10 days, the culture had reached $80-90 \%$ confluence, following trypsin digestion the third passage was used for further experiments.

Preparation of hemolysate. Hemolysate was prepared using a freeze-thaw method previously described (10), although with several modifications, to lyse red blood cells. A heparinized sterile syringe was used to collect $1 \mathrm{ml}$ blood from the rat tail artery. The blood was transferred to a sterile centrifuge tube, and then centrifuged at 2,500 $\mathrm{x} g$ for $15 \mathrm{~min}$ at $4^{\circ} \mathrm{C}$. The serum was discarded, and the red blood cells were resuspended in sterile distilled water. The cells were frozen at $-20^{\circ} \mathrm{C}$ for $20 \mathrm{~min}$ and then immediately transferred to a $39^{\circ} \mathrm{C}$ water bath. After the cells had completely thawed, they were centrifuged at $12,000 \times \mathrm{g}$ for $30 \mathrm{~min}$ at $4{ }^{\circ} \mathrm{C}$. The supernatant containing the hemolysate was collected. Hemolysate concentration was calculated as follows: Concentration $=($ hemolysate mass - distilled water mass) / hemolysate volume. All procedures were performed under sterile conditions.

Treatment of cells. Certain of these experiments included 2-[(4-phenoxyphenylsulfonyl)methyl]-thiirane (SB-3CT), a selective MMP-9 inhibitor purchased from Sigma-Aldrich; Merck Millipore. As shown in Fig. 1A, the cells were randomly assigned into three groups: i) The control group, where cerebrovascular smooth muscle cells were cultured under normal conditions; ii) the hemolysate treatment group, where the cells were treated with $1 \mathrm{mg} / \mathrm{ml}$ hemolysate for $24 \mathrm{~h}$; and iii) the SB-3CT treatment group, where cells were pretreated with $1 \mu \mathrm{M}$ SB-3CT for $6 \mathrm{~h}$, and subsequently treated with $1 \mathrm{mg} / \mathrm{ml}$ hemolysate for a further $24 \mathrm{~h}$.

Western blot analysis. Following treatment, the culture medium was discarded and cells were rinsed three times with phosphate-buffered saline (PBS). Cell lysis buffer (Beyotime Institute of Biotechnology, Haimen, China) was added and the cells were scraped off the dishes and maintained on ice. Cell lysates were placed on ice for $30 \mathrm{~min}$ prior to centrifugation at $12,000 \mathrm{x} g$ for $10 \mathrm{~min}$ at $4^{\circ} \mathrm{C}$. Protein concentration was determined using the bicinchoninic acid assay (BCA) method. The cell lysates were transferred to a 96-well plate, and $200 \mu \mathrm{l}$ BCA working solution was added to each well. After a $30 \mathrm{~min}$ incubation at $37^{\circ} \mathrm{C}$, the absorbance at $562 \mathrm{~nm}$ was determined using a microplate reader. A standard curve was generated and used to determine the concentrations of the samples. The samples were then separated using sodium dodecyl sulfate-polyacrylamide gel electrophoresis (10\% gels), and transferred to a nitrocellulose membrane. The membranes were blocked with $1 \mathrm{X}$ PBS-Tween 20 (PBST) solution with 5\% bovine serum albumin (BSA; Beyotime Institute of Biotechnology) for $1 \mathrm{~h}$ at room temperature. The membranes were then incubated with primary antibodies against MMP-9 (cat. no. ab119906), type IV collagen (cat. no. ab19808), type V collagen (cat. no. ab114072) and $\beta$-actin (cat. no. ab8227; all at a dilution of 1:1,000, and obtained from Abcam, Cambridge, MA, USA) overnight at $4^{\circ} \mathrm{C}$. The membranes were rinsed three times with PBST ( 5 min per rinse) and then incubated with a horseradish peroxidase-conjugated secondary antibodies (cat. nos. ab6789; ab 6721) at 1:5,000 dilution for $2 \mathrm{~h}$ at room temperature. Finally, the membranes were rinsed with PBST three times (5 min per rinse) and visualized using Pierce ECL Western Blotting substrate (cat. no. 32106; Thermo Fisher Scientific, Inc.).

Immunofluorescence staining. Cover slides were placed in 12 -well plates, coated with $0.1 \%$ poly-lysine (Sigma-Aldrich, Merck Millipore) overnight and rinsed three times with sterile distilled water. Cells were seeded into 12 -well plates at a density of $1.0 \times 10^{5}$ cells/well, cultured until they reached $70 \%$ confluence, and then treated with hemolysate and SB-3CT, as described above. Following the treatment, the medium was removed and the cells were rinsed three times with PBS and fixed in $4 \%$ paraformaldehyde for $20 \mathrm{~min}$. The cells were rinsed with PBS and blocked with PBS with 5\% BSA for $30 \mathrm{~min}$ at room temperature. Following blocking, the cells were incubated with a 1:1,000 dilution of primary antibody against $\alpha$-smooth muscle actin ( $\alpha$-SMA; a marker for cerebrovascular smooth muscle cells; cat. no. ab7817; Abcam), MMP-9, type IV and $\mathrm{V}$ collagen (Abcam) overnight at $4^{\circ} \mathrm{C}$. The cells were then washed three times with PBS and incubated with fluorescent, labeled secondary antibodies (cat nos. ab150077; ab150115; Abcam) at room temperature for $30 \mathrm{~min}$. Following three additional washes using PBS, the cell nuclei were stained using 4',6-diamidino-2-phenylindole. The slides were then sealed and observed under a BX50/BX-FLA/DP70 fluorescence microscope (Olympus Corporation, Tokyo, Japan).

Collagen gel contraction assay. Cell contraction assay kits were purchased from Cell Biolabs, Inc. (San Diego, CA, USA) and used according to the manufacturer's protocol. Following 
A

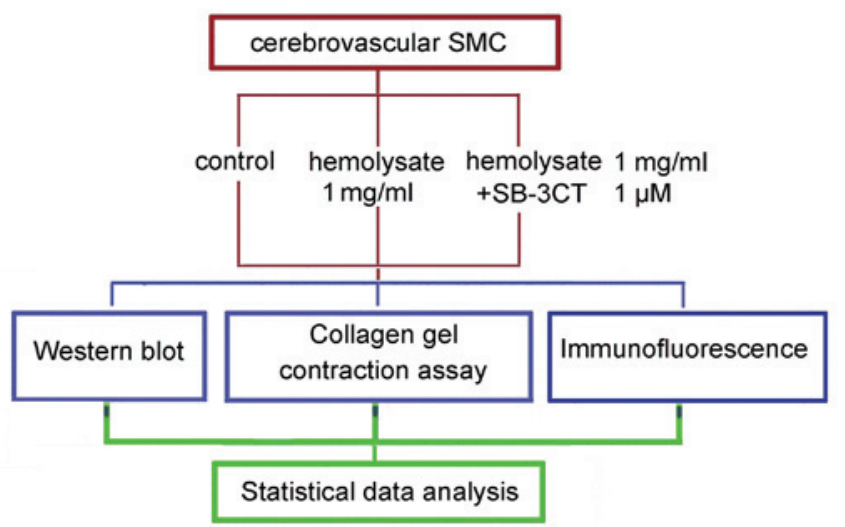

B
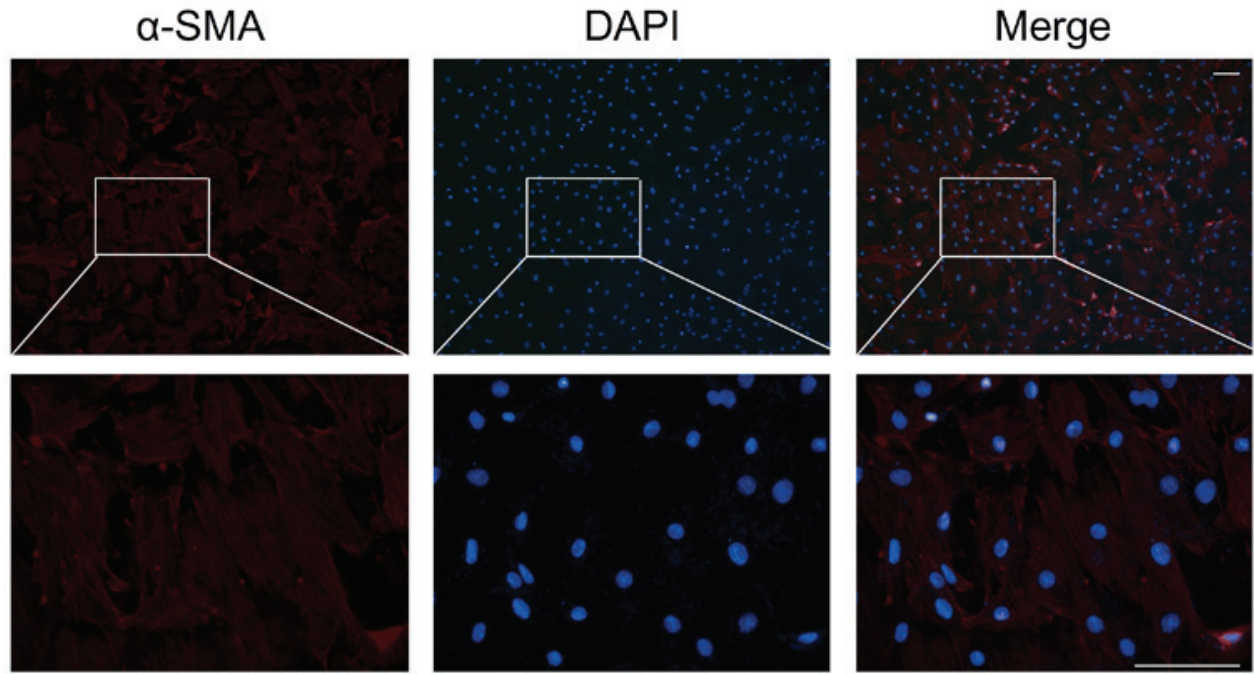

Figure 1. Experimental design and identification of cerebrovascular SMC. (A) Experiments were designed to detect changes in expression levels of MMP-9, collagen IV and collagen V in the cerebrovascular SMC following hemolysate treatment. (B) Identification of cerebrovascular SMC. Red fluorescence indicates staining for $\alpha$-SMA, which is a specific marker for cerebrovascular SMC. Blue fluorescence indicates nuclei stained with DAPI. Magnification of the upper panels, x100; that of the lower panels, x400. Scale bar, $50 \mu \mathrm{m}$. SMC, smooth muscle cells; SB-3CT, 2-[(4-phenoxyphenylsulfonyl)methyl]thiirane; $\alpha$-SMA, $\alpha$-smooth muscle actin; DAPI, 4',6-diamidino-2-phenylindole; MMP-9, matrix metalloproteinase 9.

treatment (Fig. 1), cells were trypsinized and suspended, rinsed three times with PBS, centrifuged at 1,500 $\mathrm{x}$ for $3 \mathrm{~min}$ and resuspended in phenol red-free DMEM (Thermo Fisher Scientific, Inc.) at a final concentration of $5.0 \times 10^{6}$ cells $/ \mathrm{ml}$. Rat tail collagen solution (Gibco; Thermo Fisher Scientific, Inc.) was diluted to a final concentration of $3 \mathrm{mg} / \mathrm{ml}$ with $0.3 \mathrm{ml}$ phenol red-free DMEM ( $\mathrm{pH}$ 7.3-7.4). Collagen solution, cell suspension and FBS were then combined at a ratio of 8:1:1 to yield a final concentration of $5.0 \times 10^{5}$ cells $/ \mathrm{ml}$. Aliquots $(1 \mathrm{ml} /$ well $)$ of the collagen-cell mixture were added to a $24-$ well plate and the plates were incubated for $30 \mathrm{~min}$ at $37^{\circ} \mathrm{C}$ with $5 \% \mathrm{CO}_{2}$ to promote polymerization. Following the solidification of the gel, the edges of the gel attached to the wells were scored using pipette tips and an appropriate quantity of phenol red-free DMEM was added. Gels were incubated for an additional $24 \mathrm{~h}$ prior to image capture. Cell contractility was calculated using the following formula: Contraction index $=($ well area - gel area $) /$ well area $\mathrm{x} 100 \%$.

Statistical analysis. The western blotting and immunofluorescent staining were analyzed using ImageJ version 1.46 software (National Institutes of Health, Bethesda, MD, USA. The mean grayscale value of the control group was normalized to 1 , and the ratios of the grayscale values of the experimental groups to the control group were calculated and analyzed. Statistical analysis was performed using GraphPad Prism version 5.0 software (GraphPad Software, Inc., La Jolla, CA, USA). Data are presented as the mean \pm standard error. One-way analysis of variance and Fisher's least significant difference test was used for pairwise comparison of groups. $\mathrm{P}<0.05$ was considered to indicate a statistically significant difference.

\section{Results}

Isolation and culture of cerebrovascular smooth muscle cells. Immunofluorescence staining was performed to determine the phenotype of the adherent cells. As presented in Fig. 1B, the rat cerebrovascular smooth muscle cells had a polygonal or long, spindle-like morphology and expanded into a monolayer when observed under a microscope. It was demonstrated that the majority of isolated cells stained positively for $\alpha$-SMA, a specific marker of cerebrovascular smooth muscle cells. This indicated that the cerebrovascular smooth muscle cells had been successfully isolated (Fig. 1B).

Hemolysate enhances the contractility of cerebrovascular smooth muscle cells. As presented in Fig. 2, the contractility of 
A control

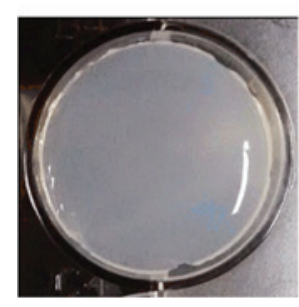

hemolysate

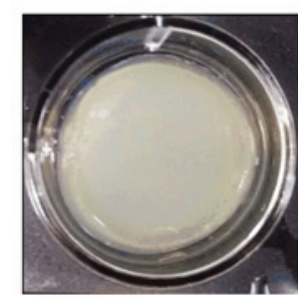

B

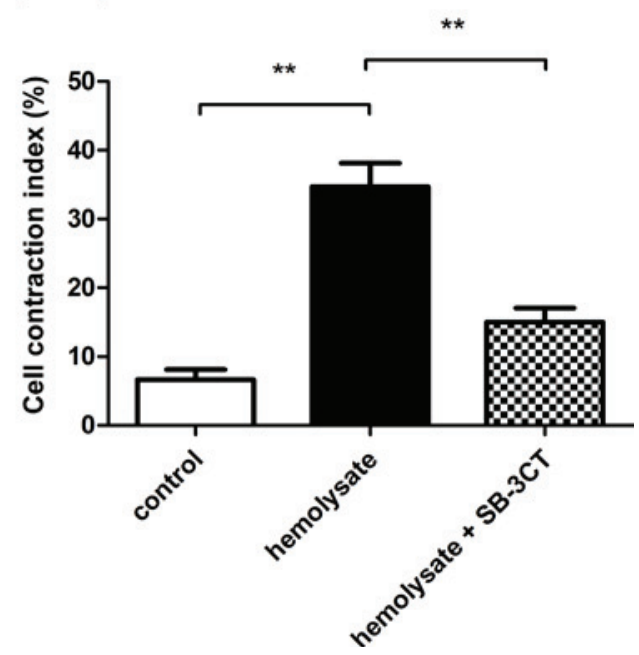

Figure 2. Hemolysate enhances the contractility of cerebrovascular smooth muscle cells. (A) Images of gel contraction following treatment with $1 \mathrm{mg} / \mathrm{ml}$ hemolysate and (B) quantification of the gel contraction. Data were derived from three independent experiments. ${ }^{* *} \mathrm{P}<0.01 \mathrm{vs}$. control group. SB-3CT, 2-[(4-phenoxyphenylsulfonyl)methyl]thiirane.

A

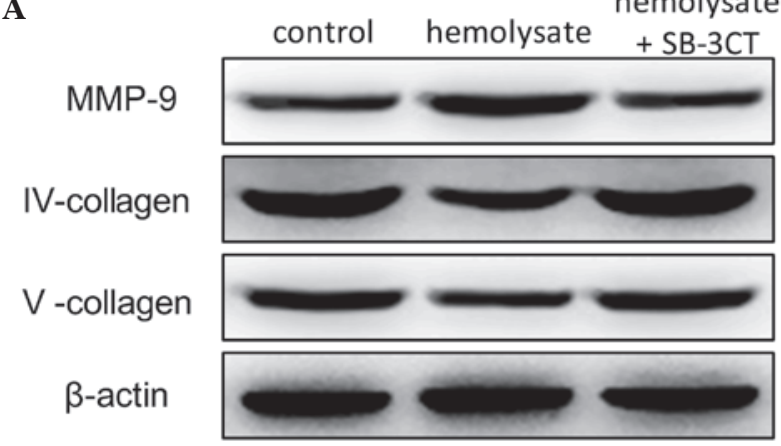

B

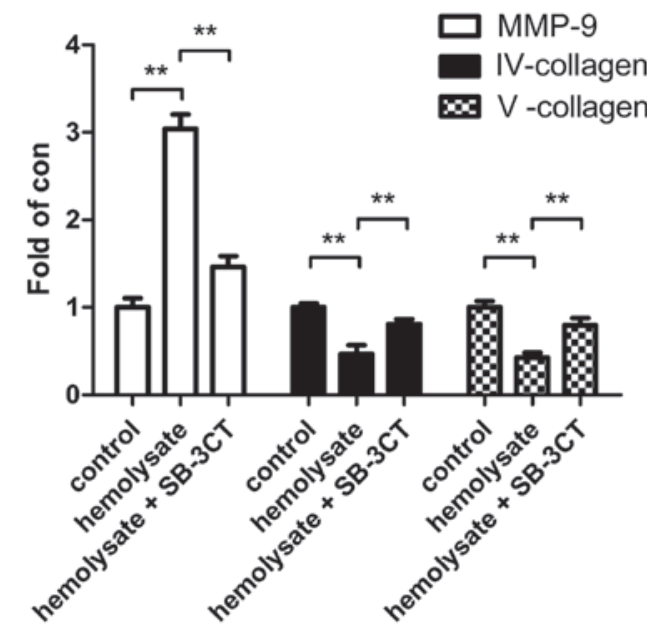

Figure 3. Western blot analysis demonstrated that hemolysate upregulates MMP-9 expression in cerebrovascular smooth muscle cells. (A) Western blot analysis of MMP-9 and collagen IV and V. (B) Quantification of the western blotting. Data were derived from three independent experiments. ${ }^{* *} \mathrm{P}<0.01$ vs. control group or the hemolysate + SB-3CT group. MMP-9, matrix metallopeptidase 9; SB-3CT, 2-[(4-phenoxyphenylsulfonyl) methyl]thiirane.

cerebrovascular smooth muscle cells was determined using a collagen gel contraction assay. Significant gel contraction was observed in the hemolysate treatment group when compared with the control group ( $\mathrm{P}<0.01$; Fig 2B). However, pretreatment with SB-3CT significantly inhibited the hemolysate-induced gel contraction when compared with the hemolysate treatment group $(\mathrm{P}<0.01$; Fig. $2 \mathrm{~B})$.

Hemolysate increases MMP-9 protein expression levels in cerebrovascular smooth muscle cells. Western blot analysis was used to demonstrate that hemolysate stimulation significantly increased MMP-9 protein expression levels $(\mathrm{P}<0.01$; Fig. 3) and reduced collagen IV and V protein expression levels $(\mathrm{P}<0.01$; Fig. 3B) when the hemolysate treatment group was compared with the control. However, pretreatment of cerebrovascular smooth muscle cells with SB-3CT significantly reduced the expression levels of MMP-9 when compared with the hemolysate treatment group $(\mathrm{P}<0.01$; Fig. 3B). Conversely, collagen IV and $\mathrm{V}$ protein expression levels were significantly greater in the SB-3CT pretreatment group compared with the hemolysate group $(\mathrm{P}<0.01$; Fig. $3 \mathrm{~B})$.

Immunofluorescence staining also demonstrated that hemolysate treatment significantly increased the protein expression levels of MMP-9 ( $\mathrm{P}<0.01$; Fig. 4) and reduced the protein expression levels of collagens IV and $\mathrm{V}(\mathrm{P}<0.01$; Fig. 4) in cerebrovascular smooth muscle cells compared with the control group. However, pretreatment with SB-3CT significantly reduced the MMP-9 expression levels ( $\mathrm{P}<0.01$; Fig. 4D). Collagen IV and $\mathrm{V}$ protein expression levels were significantly increased in the SB-3CT pretreatment group compared with the hemolysate group $(\mathrm{P}<0.01$; Fig. 4).

\section{Discussion}

Intracranial aneurysm is a common cerebrovascular disease. Aneurysm re-rupture and CVS are two major complications that may occur following a ruptured aneurysm. CVS occurs at a rate of $30-70 \%$, with $\sim 30 \%$ of these patients presenting symptoms of cerebral ischemia or severe cerebral infarctions (1). As the pathogenesis of CVS remains to be fully elucidated, the treatment options are currently limited. Therefore, prevention of CVS is crucial for the reduction of 
A
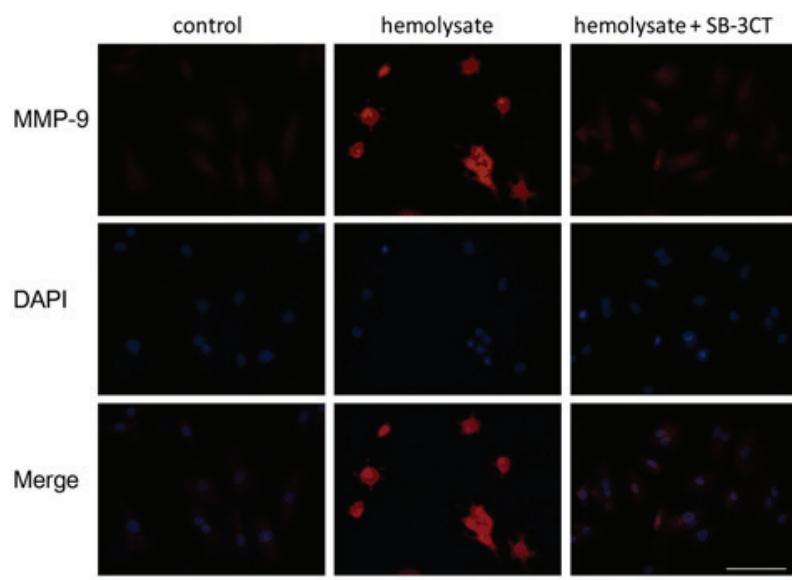

C
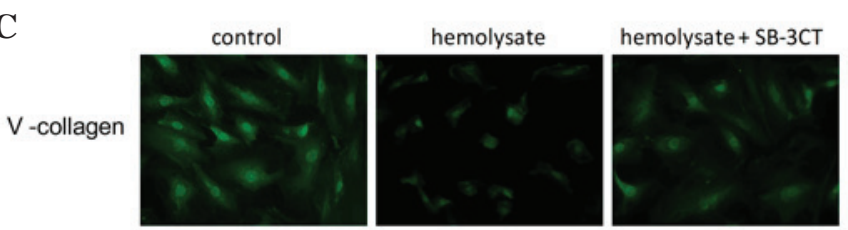

DAPI
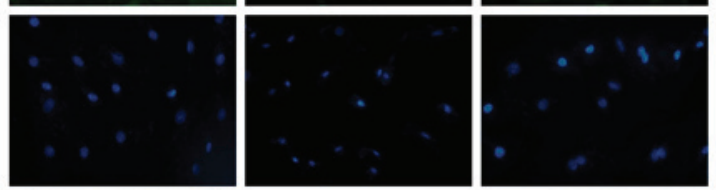

Merge
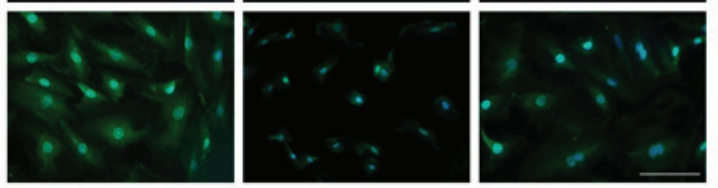
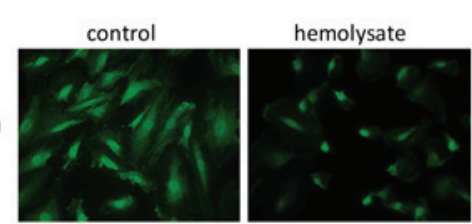

hemolysate + SB-3CT
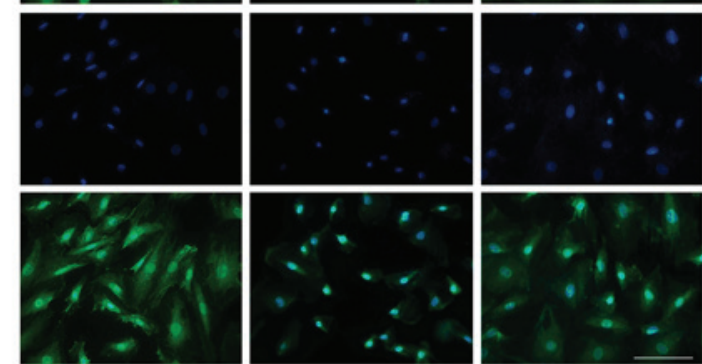

D

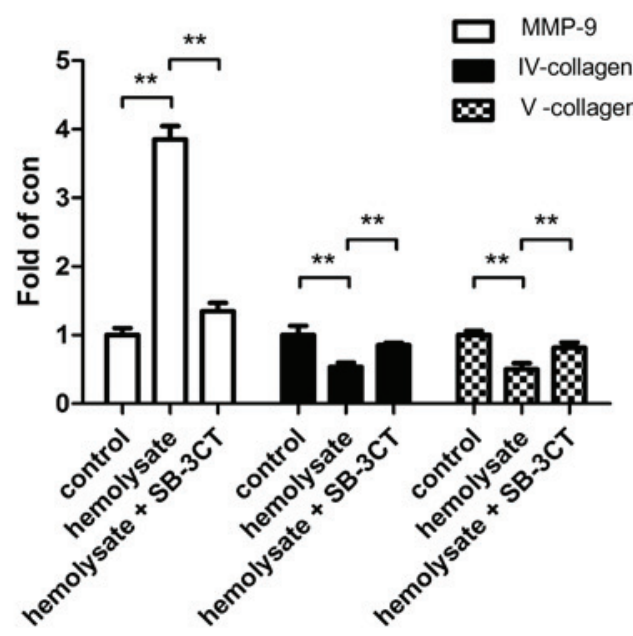

Figure 4. Immunofluorescent staining demonstrated that hemolysate upregulates the epxression levels of MMP-9 in cerebrovascular smooth muscle cells. Immunofluorescent staining for (A) MMP-9, (B) collagen IV and (C) collagen V following treatment with $1 \mathrm{mg} / \mathrm{ml}$ hemolysate with and without pretreatment using $1 \mu \mathrm{M}$ SB-3CT. Magnification, $\mathrm{x} 400$. Scale bar, $50 \mu \mathrm{m}$. (D) Quantification of immunofluorescent staining. Data were derived from three independent experiments. ${ }^{* *} \mathrm{P}<0.01$ vs. control group or the hemolysate + SB-3CT group. MMP-9, matrix metallopeptidase 9; SB-3CT, 2-[(4-phenoxyphenylsulfonyl)methyl] thiirane; DAPI, 4',6-diamidino-2-phenylindole.

morbidity and mortality rates following intracranial aneurysm surgery.

Previous studies have determined that CVS may be due to multiple factors that enhance cerebral vasoconstriction $(2,4)$ and diminish cerebral vasodilation $(3,5,11)$. Humphrey et al (11) proposed a theoretical biomechanical framework of SAH vasospasm (11), which noted that, following SAH, extracellular clot stimulation of blood vessel walls led to increased levels of NO scavengers, such as reactive oxygen species and oxyhemoglobin, including higher levels of serotonin, thromboxane A2, angiotensin-1 and thrombin. This, in turn, may lead to increased vascular thickness and hardness, stenosis and vasoconstriction. The vessel may return to normal when the clot dissolves. Different treatment strategies have been evaluated based on these mechanisms. Previous studies have determined the effects of several antagonists on the contraction of cerebral smooth muscle, including calcium channel blockers such as papaverine (12) and nicardipine (13). Fenpropathrin povidone (14) and endothelin antagonists (15) were also revealed to have a therapeutic effect. However, the overall effectiveness of these treatments was unsatisfactory as of the exact pathogenesis of remains to be elucidated.

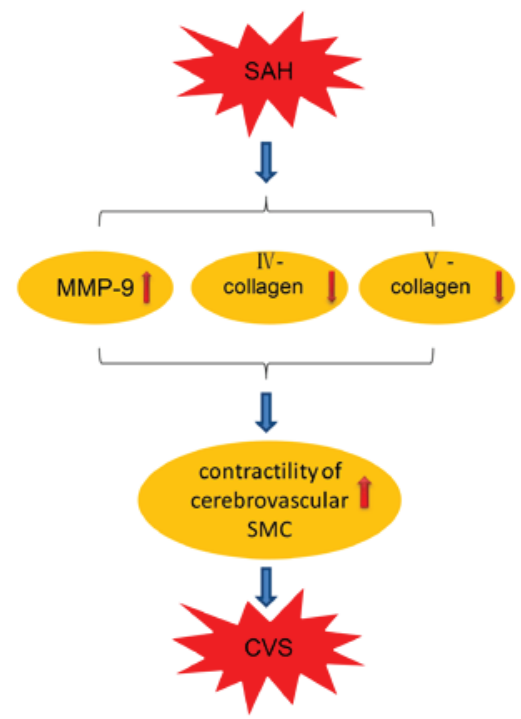

Figure 5. Hypothesized model for the importance of MMP-9 in cerebrovascular SMC following SAH. MMP-9 expression levels were increased in cerebrovascular SMC, whereas expression levels of collagen IV and V decreased significantly following $\mathrm{SAH}$. These changes led to enhanced contractility in cerebrovascular SMC and led to CVS. SAH, subarachnoid hemorrhage; MMP-9, matrix metallopeptidase 9; SMC, smooth muscle cells; CVS, cerebral vasospasm. 
Previous studies have determined that MMP-9 may be associated with cerebrovascular disease. MMP-9 may degrade the extracellular matrix (16-21). Additionally, it may be involved in various pathological processes, including the degradation of the connective tissue, inflammation, ischemia and hypoxia. Therefore, MMP-9 is important for pathological processes associated with cerebral hemorrhage. Compared with previous studies on cerebral hemorrhage, the effects of MMP-9 on aneurysmal SAH remain to be fully elucidated. However, the current study identified MMP-9 as a potential biomarker for CVS following an aneurysm (16). Previous studies have determined that, following aneurysmal SAH, the expression levels of MMP-9 are increased in brain tissue, cerebrospinal fluid and peripheral blood (17-19). Therefore, MMP-9 is important in the pathological processes of aneurysmal SAH $(17,20,21)$.

Our previous study on a rat model revealed that CVS may occurs following SAH (9). Additionally, protein expression levels of MMP-9 in the basilar artery walls increased following SAH with a parallel time course to development of CVS. This suggested that MMP-9 may be involved in the pathological processes of CVS (9). CVS primarily occurs due to enhanced contractility of cerebrovascular smooth muscle cells. The aim of the present study was to determine whether MMP-9 may be involved in this process (Fig. 5). Cerebrovascular smooth muscle cells were treated with hemolysate as an in vitro model of SAH. Collagen gel contraction experiments revealed that hemolysate treatment induced a significant contractile response in cerebrovascular smooth muscle cells. In addition, pretreatment with SB-3CT (a selective inhibitor of MMP-9) reduced these contractile responses. Immunofluorescence staining and western blotting confirmed that hemolysate stimulation increased MMP-9 protein expression levels in cerebrovascular smooth muscle cells. In contrast, protein expression levels of collagen IV and $\mathrm{V}$ were significantly decreased. This suggests that MMP-9 contributed to protease activity, catalyzed the degradation of collagen IV and V and was important for the contractile response of cerebrovascular smooth muscle cells. Additionally, pretreatment with SB-3CT inhibited the protease activity of MMP-9 and the contractile responses of the cerebrovascular smooth muscle cells.

In conclusion, the present study used an in vitro model of SAH to investigate changes in MMP-9 expression levels in cerebrovascular smooth muscle cells, and its involvement in their contractile response. Therefore, the current study provided novel insights into the pathogenesis of CVS following SAH. Further research is necessary to elucidate the specific molecular mechanisms that mediate the effects of MMP-9 on the contraction of cerebrovascular smooth muscle cells, and whether MMP-9 is involved in cerebral inflammation following SAH.

\section{Acknowledgements}

The present study was supported by grants from the Suzhou Government (grant nos. KJXW2014042 and ZKS1419).

\section{References}

1. Baggott CD and Aagaard-Kienitz B: Cerebral vasospasm. Neurosurg Clin N Am 25: 497-528, 2014.

2. Nishizawa $S$ and Laher I: Signaling mechanisms in cerebral vasospasm. Trends Cardiovasc Med 15: 24-34, 2005.

3. Pluta RM: Delayed cerebral vasospasm and nitric oxide: Review, new hypothesis and proposed treatment. Pharmacol Ther 105: 23-56, 2005.

4. Sehba FA and Bederson JB: Mechanisms of acute brain injury after subarachnoid hemorrhage. Neurol Res 28: 381-398, 2006.

5. Suhardja A: Mechanisms of disease: Roles of nitric oxide and endothelin-1 in delayed cerebral vasospasm produced by aneurysmal subarachnoid hemorrhage. Nat Clin Pract Cardiovasc Med 1: 110-116; quiz $2 \mathrm{p}$ following 116, 2004.

6. Cunningham LA, Wetzel M and Rosenberg GA: Multiple roles for MMPs and TIMPs in cerebral ischemia. Glia 50: 329-339, 2005.

7. Fatar M, Stroick M, Griebe M and Hennerici M: Matrix metalloproteinases in cerebrovascular diseases. Cerebrovasc Dis 20: 141-151, 2005.

8. Rosenberg GA: Matrix metalloproteinases in neuroinflammation. Glia 39: 279-291, 2002.

9. Wang Z, Fang Q, Dang BQ, Shen XM, Shu Z, Zuo G, He WC and Chen G: Potential contribution of matrix metalloproteinase-9 (mmp-9) to cerebral vasospasm after experimental subarachnoid hemorrhage in rats. Ann Clin Lab Sci 42: 14-20, 2012.

10. Choudhri TF, Hoh BL, Solomon RA, Connolly ES Jr and Pinsky DJ: Use of a spectrophotometric hemoglobin assay to objectively quantify intracerebral hemorrhage in mice. Stroke 28: 2296-2302, 1997.

11. Humphrey JD, Baek S and Niklason LE: Biochemomechanics of cerebral vasospasm and its resolution: I. A new hypothesis and theoretical framework. Ann Biomed Eng 35: 1485-1497, 2007.

12. Kim JH, Yi HJ, Ko Y, Kim YS, Kim DW and Kim JM: Effectiveness of papaverine cisternal irrigation for cerebral vasospasm after aneurysmal subarachnoid hemorrhage and measurement of biomarkers. Neurol Sci 35: 715-722, 2014.

13. Kasuya H, Onda H, Takeshita M, Okada Y and Hori T: Efficacy and safety of nicardipine prolonged-release implants for preventing vasospasm in humans. Stroke 33: 1011-1015, 2002.

14. Nishiguchi M, Ono S, Iseda K, Manabe H, Hishikawa T and Date I: Effect of vasodilation by milrinone, a phosphodiesterase III inhibitor, on vasospastic arteries after a subarachnoid hemorrhage in vitro and in vivo: Effectiveness of cisternal injection of milrinone. Neurosurgery 66: 158-164; discussion 164, 2010.

15. Macdonald RL, Higashida RT, Keller E, Mayer SA, Molyneux A, Raabe A, Vajkoczy P, Wanke I, Bach D, Frey A, et al: Randomized trial of clazosentan in patients with aneurysmal subarachnoid hemorrhage undergoing endovascular coiling. Stroke 43: 1463-1469, 2012.

16. Zhang Y, Clark JF, Pyne-Geithman G and Caruso J: Metallomics study in CSF for putative biomarkers to predict cerebral vasospasm. Metallomics 2: 628-637, 2010.

17. Horstmann S, Su Y, Koziol J, Meyding-Lamadé U, Nagel S and Wagner S: MMP-2 and MMP-9 levels in peripheral blood after subarachnoid hemorrhage. J Neurol Sci 251: 82-86, 2006.

18. Rosell A, Ortega-Aznar A, Alvarez-Sabín J, Fernández-Cadenas I, Ribó M, Molina CA, Lo EH and Montaner J: Increased brain expression of matrix metalloproteinase- 9 after ischemic and hemorrhagic human stroke. Stroke 37: 1399-1406, 2006.

19. Sarrafzadeh A, Copin JC, Bengualid DJ, Turck N, Vajkoczy P, Bijlenga P, Schaller K and Gasche Y: Matrix metalloproteinase-9 concentration in the cerebral extracellular fluid of patients during the acute phase of aneurysmal subarachnoid hemorrhage. Neurol Res 34: 455-461, 2012.

20. Chou SH, Feske SK, Simmons SL, Konigsberg RG, Orzell SC, Marckmann A, Bourget G, Bauer DJ, De Jager PL, Du R, et al: Elevated peripheral neutrophils and matrix metalloproteinase 9 as biomarkers of functional outcome following subarachnoid hemorrhage. Transl Stroke Res 2: 600-607, 2011.

21. McGirt MJ, Lynch JR, Blessing R, Warner DS, Friedman AH and Laskowitz DT: Serum von Willebrand factor, matrix metalloproteinase-9, and vascular endothelial growth factor levels predict the onset of cerebral vasospasm after aneurysmal subarachnoid hemorrhage. Neurosurgery 51: 1128-1134; discussion 1134-1135, 2002. 